TRANSACTIONS OF THE

AMERICAN MATHEMATICAL SOCIETY

Volume 350, Number 11, November 1998, Pages 4553-4568

S $0002-9947(98) 02340-\mathrm{X}$

\title{
CONSEQUENCES OF CONTRACTIBLE GEODESICS ON SURFACES
}

\author{
J. DENVIR AND R. S. MACKAY
}

\begin{abstract}
The geodesic flow of any Riemannian metric on a geodesically convex surface of negative Euler characteristic is shown to be semi-equivalent to that of any hyperbolic metric on a homeomorphic surface for which the boundary (if any) is geodesic. This has interesting corollaries. For example, it implies chaotic dynamics for geodesic flows on a torus with a simple contractible closed geodesic, and for geodesic flows on a sphere with three simple closed geodesics bounding disjoint discs.
\end{abstract}

\section{InTRODUCTION}

There is a growing number of results of the form that certain simple topological hypotheses on a dynamical system force complicated behaviour. Perhaps the best known are Sharkovskii's theorem [Sh] and "Period 3 implies chaos" [LY], for endomorphisms of the interval. More recently, analogous results have been obtained for two-dimensional diffeomorphisms: certain "braid types" (a conjugacy class of the isotopy class of the diffeomorphism relative to a finite invariant set) imply complicated dynamics (for a review see [Boy]).

In the present paper, this type of result is extended to geodesic flows on surfaces. We denote the geodesic flow of a Riemannian metric $\rho$ on a surface $M$ by $\left(\phi^{\rho}, S^{\rho} M\right)$; it is the continuous action of $(\mathbb{R},+)$ on the unit tangent bundle $S^{\rho} M$ given by following the geodesics at unit speed. Surfaces with boundary are permitted, in which case we require the surface to be geodesically convex, i.e. there are no geodesic segments $\gamma:(-\epsilon, \epsilon) \rightarrow M$ such that $\gamma(0) \in \partial M$ and $\gamma(t) \in M-\partial M$ for some $t \in(-\epsilon, \epsilon)$; in this case the flow ceases to be defined when geodesics reach the boundary. We denote by $Z^{\rho}$ the subset of $S^{\rho} M$ for which the forward and backward orbits never reach the boundary $\left(Z^{\rho}\right.$ is the whole of $S^{\rho} M$ in the case without boundary).

It is well-known that compact surfaces of negative Euler characteristic support metrics of constant negative curvature, which we call "hyperbolic", and that the dynamics of the geodesic flow of a hyperbolic metric $h$ is complicated, being equivalent on $Z^{h}$ to a suspension of a subshift of finite type (or a mild quotient of such in the case of a surface without boundary). The study of geodesic flows on surfaces with hyperbolic metric dates back to the late part of the $19^{\text {th }}$ century and the work of Hadamard. The techniques of coding such geodesics by sequences of symbols were developed by Morse and Koebe. For more details about this, see [S2].

Received by the editors September 7, 1995 and, in revised form, September 13, 1996.

1991 Mathematics Subject Classification. Primary 58F17.

Key words and phrases. Geodesics, semiconjugacy, surface, dynamics.

(C)1998 American Mathematical Society 
We will prove that the flow of any metric on a surface is at least as complicated as for a hyperbolic metric on that surface. To make this precise we introduce the following definition.

Definition 1. A flow $(\phi, X)$ is semi-equivalent to a flow $(\psi, Y)$ if there exists a continuous surjection $\Theta: X \rightarrow Y$ and continuous map $\tau: X \times \mathbb{R} \rightarrow \mathbb{R}$ such that (i) $\tau(x, \cdot)$ is an orientation-preserving homeomorphism of $\mathbb{R}$ for each $x \in X$, (ii) $\Theta \circ \phi_{t}(x)=\psi_{\tau(x, t)} \circ \Theta(x)$ for all $x \in X, t \in \mathbb{R}$, and (iii) $\tau\left(\phi_{s}(x), t\right)=\tau(x, s+t$ ) for all $s, t \in \mathbb{R}$ and all $x \in X$.

Our main result is

Theorem 2. Given a Riemannian metric $\rho$ on a compact surface $M$ of negative Euler characteristic, with or without boundary, which is geodesically convex, and a hyperbolic metric $h$ on a homeomorphic surface for which the boundary is geodesic, there exists a $\phi^{\rho}$-invariant subset $\mathcal{A} \subset S^{\rho} M$ and a semi-equivalence $(\Theta, \tau)$ from $\left(\phi^{\rho}, \mathcal{A}\right)$ to $\left(\phi^{h}, Z^{h}\right)$.

In the case without boundary, this theorem is a relatively simple consequence of a theorem of Morse [M1]. So our main contribution is to prove it in the case with boundary. Nevertheless, we also give a new proof in the case without boundary, as we find our approach much cleaner than Morse's.

The key idea from Morse is to study the "class A" geodesics. A geodesic is "class A" if it is doubly infinite and minimises the length between any two of its points with respect to homotopy. For a hyperbolic metric, all doubly infinite geodesics are class A. For a general metric, each class A geodesic $\gamma$ is "globally shadowed" by a unique hyperbolic geodesic $\hat{\gamma}$, meaning that on the universal cover $\hat{\gamma}$ remains within a bounded distance of $\gamma$. Furthermore, for each hyperbolic geodesic there exists a globally shadowing class A geodesic of the general metric. Morse proved these results in the case without boundary and we prove them in the case with boundary. Then we define $\mathcal{A}$ to be the unit tangents to the class A geodesics, and a simple argument leads to the semi-equivalence. Our main new idea is to prove the global shadowing by making a careful choice of fundamental domain for the universal cover.

As Corollaries of Theorem 2, we deduce complicated dynamics for certain geodesic flows on the sphere and torus, despite the fact that these surfaces do not have negative Euler characteristic.

Corollary 3. Given a Riemannian metric on a torus with a simple, closed contractible geodesic, there is an invariant subset on which the geodesic flow is semiequivalent to that of a hyperbolic metric on the torus with a disk removed, restricted to its doubly infinite geodesics.

Corollary 4. Given a Riemannian metric on a sphere with three simple, closed geodesics bounding disjoint disks, there is an invariant subset on which the geodesic flow is semi-equivalent to that of a hyperbolic metric on the sphere with three disjoint disks removed, restricted to its doubly infinite geodesics.

Proofs. Simply remove the disks bounded by the given geodesics and apply Theorem 2.

Remark 5. Hyperbolic metrics. All hyperbolic metrics on a given surface have equivalent geodesic flows (where equivalence of flows is defined as for semi-equival- 
ence, but with $\Theta$ a homeomorphism). Thus it is not necessary to specify which hyperbolic metric we use.

Remark 6 . Non-simple closed geodesics. The corollaries above can be extended to cases with non-simple contractible closed geodesics, provided there is a component of the complement which has negative Euler characteristic. Each component is automatically a geodesically convex surface.

We proceed as follows. In section 2 we establish some notation and recall basic results for geodesic flows. In sections 3,4 and 5 , we restrict attention to the case of surfaces $M$ with non-empty boundary. We describe the construction of a covering space of such $M$ with special choice of fundamental domain. We define class A geodesics and study their key properties. We then prove an analogue of Morse's Theorem and deduce Theorem 2 for this case. In section 6 we treat the case without boundary, giving a new proof of Morse's Theorem and deducing Theorem 2 in this case also. Finally, in section 7 we comment on the relations with other work and potential extensions.

\section{Notation and Review of Basic Results}

Let $M$ be a compact manifold with $\mathcal{C}^{2}$ Riemannian metric $\rho$. Let $\gamma:[a, b] \rightarrow M$ be an absolutely continuous curve, and for $a \leq p \leq q \leq b$ define $L_{\rho}(\gamma ; p, q)$ to be the length of the curve $\gamma$ from $\gamma(p)$ to $\gamma(q)$ with respect to the metric $\rho$. A minimal geodesic segment is a curve $\gamma:[a, b] \rightarrow M$ such that, for each $a \leq p \leq q \leq b$ there is no curve $\alpha:[0,1] \rightarrow M$ with $\alpha(0)=\gamma(p), \alpha(1)=\gamma(q)$ and $L_{\rho}(\alpha ; 0,1)<L_{\rho}(\gamma ; p, q)$. A geodesic is a curve $\gamma: I \rightarrow M$, where $I \subset \mathbb{R}$ is a closed interval (we allow $I=\mathbb{R}$ and semi-infinite closed intervals) such that, given any $s \in I$ there is $\epsilon>0$ such that $\left.\gamma\right|_{[s-\epsilon, s+\epsilon] \cap I}$ is a minimal geodesic segment. A minimal geodesic is a curve $\gamma: \mathbb{R} \rightarrow M$ such that $\left.\gamma\right|_{[a, b]}$ is a minimal geodesic segment for any $a \leq b \in \mathbb{R}$.

We recall the following classical results; see for example $[\mathrm{Bi}],[\mathrm{C}],[\mathrm{K}]$.

Lemma 7. Given $a, b \in M$, there is a shortest path $\alpha:[p, q] \rightarrow M$ with $\alpha(p)=a$ and $\alpha(q)=b$.

Lemma 8. In each non-trivial free homotopy class, there is a closed geodesic of minimum length. Such a geodesic will be called a minimal periodic geodesic.

Lemma 9. Let $\alpha, \beta:[a, b] \rightarrow M$ be geodesics and suppose there exists $\epsilon>0$ such that $\left.\alpha\right|_{[a, a+\epsilon]}=\left.\beta\right|_{[a, a+\epsilon]}$. Then $\alpha=\beta$.

Lemma 10. A geodesic $\alpha:[a, b] \rightarrow M$ is differentiable on $[a, b]$. In particular, a geodesic 'contains no corners.'

Lemma 11. If $\rho$ is a $\mathcal{C}^{2}$ Riemannian metric, then the geodesic flow $\phi^{\rho}$ is $\mathcal{C}^{1}$.

The Poincaré disk $\Delta$ is the open unit disk in the complex plane, with the hyperbolic metric $h$ defined by $d s^{2}=\frac{|d z|^{2}}{\left(1-|z|^{2}\right)^{2}}$.

For any metric $\rho$, denote by $d_{\rho}$ the distance function generated by $\rho$.

\section{Universal COVER AND SPECIAL FUNDAMENTAL DOMAIN FOR SURFACES WITH BOUNDARY}

Throughout Sections 3, 4 and $5, M$ will be a compact orientable surface with $\mathcal{C}^{2}$ Riemannian metric $\rho$, of genus $g \geq 0$ with $s \geq 1$ open discs removed, $2 g+s \geq 3$, 


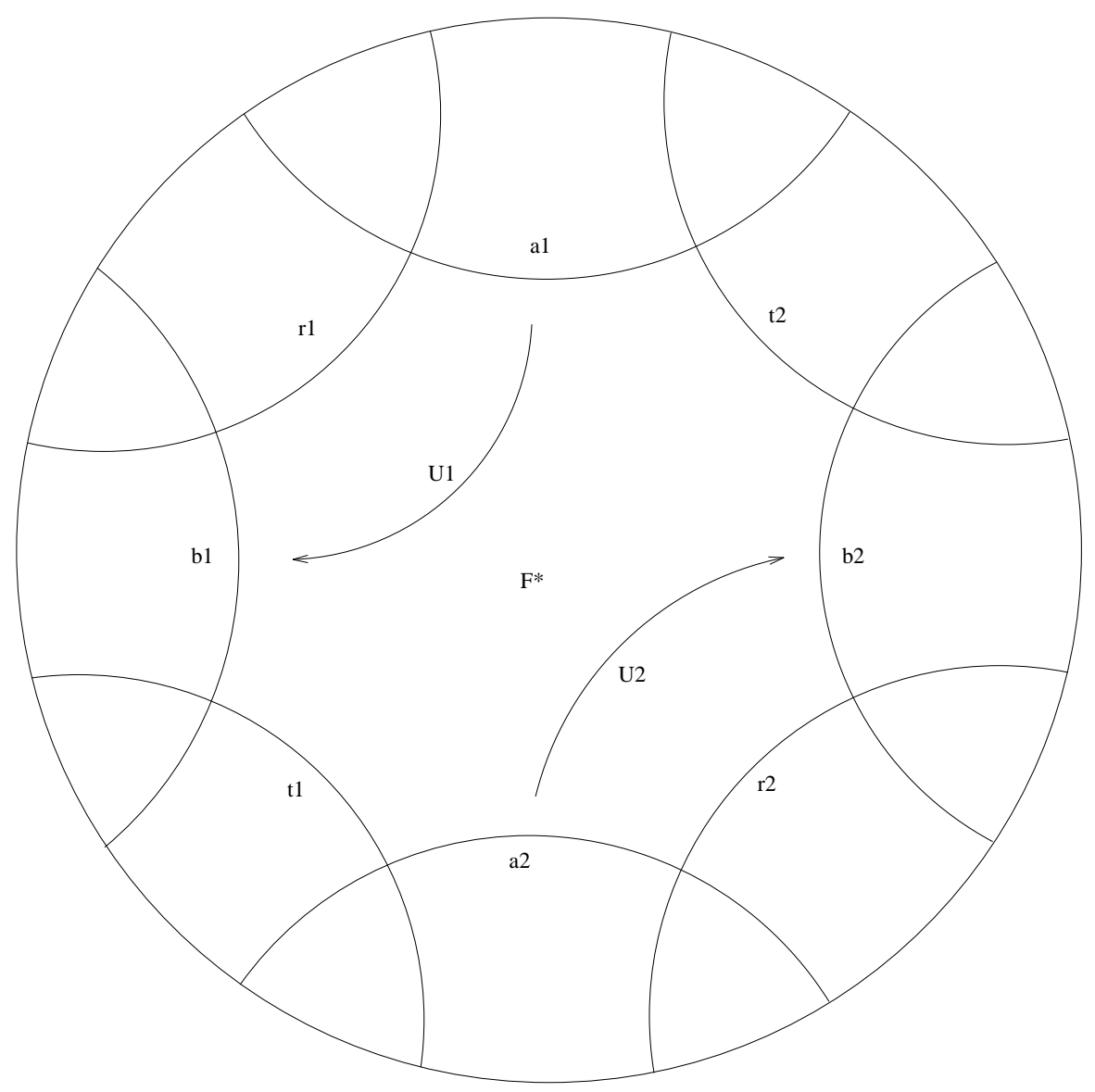

Figure 1. (case $g=0, s=3$ ).

and geodesically convex. Using a result of Bolotin [Bol1], there are disjoint simple closed geodesics around each boundary component, and so we can without loss of generality assume that each boundary component is a $\rho$-geodesic.

In this section we recall a construction of the universal covering space and fundamental group of $M$, and make a particular choice of fundamental domain. The cases of zero and positive genus are initially treated separately.

Let us first treat the case $g=0, s \geq 3$. Choose $4(s-1)$ geodesics in the Poincaré disk $\Delta$, placed with rotational symmetry about 0 , such that each intersects only its two immediate neighbours and all intersections are perpendicular. This can be achieved, because the exterior angle sum of a hyperbolic polygon is $2 \pi+A$, where $A$ is its area, and the exterior angles approach $\pi$ as the vertices approach the boundary of $\Delta$, so the exterior angle of a regular $N$-gon $(N \geq 3)$ can be varied continuously from $2 \pi / N$ to $\pi$ by increasing the area, and in particular attains the value $\pi / 2$. The geodesics continuing the boundary components of the resulting polygon intersect only at the vertices of the polygon, because two geodesics perpendicular to a given one can not intersect. Label these geodesics, in anticlockwise order, $a_{1}, r_{1}, b_{1}, t_{1}, \ldots a_{s-1}, r_{s-1}, b_{s-1}, t_{s-1}$, as in Figure 1. 


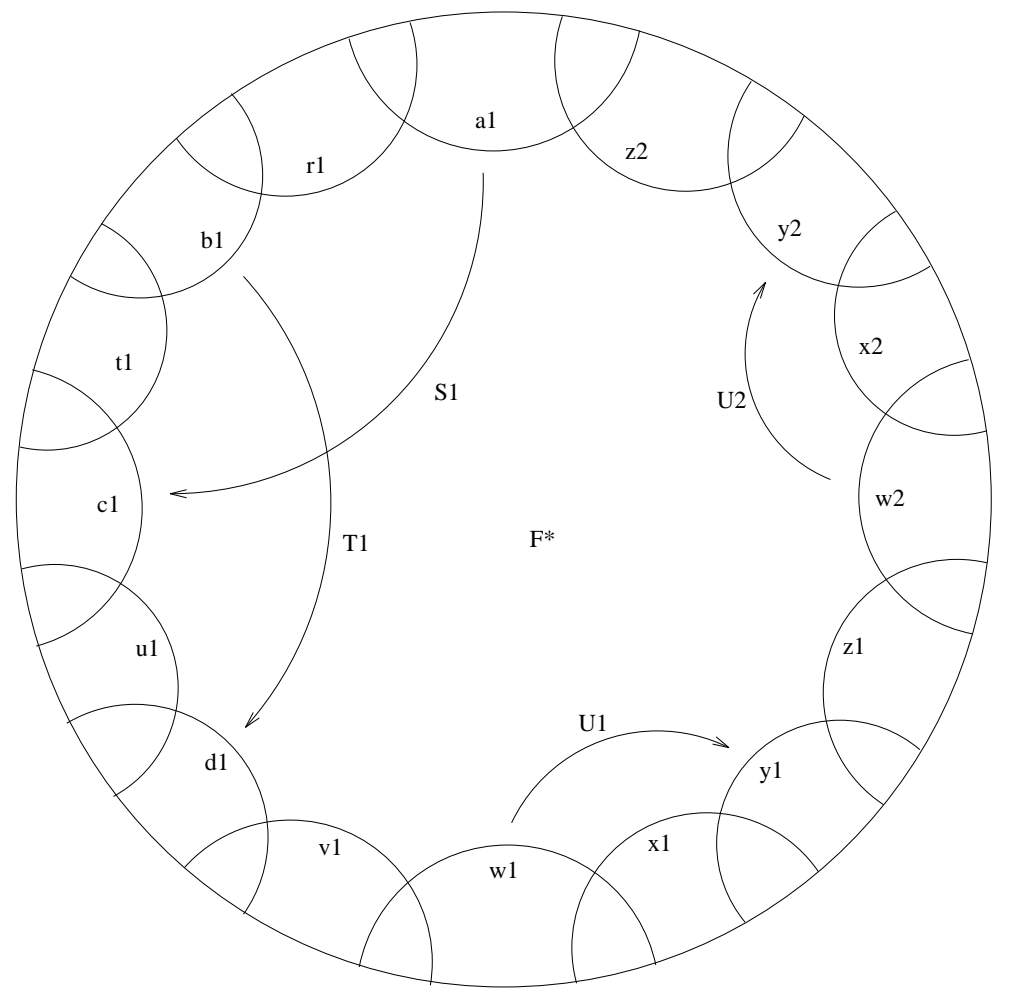

Figure 2. ( case $g=1, s=3)$.

Denote by $U_{i}$ the isometry $z \mapsto \frac{a z+\bar{c}}{c z+\bar{a}},|a|^{2}-|c|^{2}=1$, which takes the geodesic $a_{i}$ to the geodesic $b_{i}$, with $U_{i}\left(a_{i} \cap r_{i}\right)=b_{i} \cap r_{i}$ and $U_{i}\left(a_{i} \cap t_{i-1}\right)=b_{i} \cap t_{i}$ (where $\left.t_{0}=t_{s-1}\right)$. Let $G$ be the group generated by the $U_{i}$. Denote by $F^{*}$ the compact region bounded by all the $a_{i}, b_{i}, r_{i}, t_{i}$ and by $\Delta^{\prime} \subset \Delta$ the union of the images of $F^{*}$ under all the elements of $G$. Let $M^{\prime}=\Delta^{\prime} / G$ and denote by $\pi$ the natural projection $\pi: \Delta^{\prime} \rightarrow M^{\prime}$. Then each $r_{i}$ projects under $\pi$ to a boundary component of $M^{\prime}$, and $\bigcup_{i=1}^{s-1} t_{i}$ projects under $\pi$ to a further boundary component. Furthermore, $M^{\prime}$ has no handles. Thus $M^{\prime}$ is diffeomorphic to $M$.

Now let us treat the case $g \geq 1, s \geq 1$. We choose $4(2 g+s-1) \geq 8$ geodesics in the Poincaré disk, symmetrically placed about 0 , and again such that each intersects only its two immediate neighbours, with all intersections perpendicular. We label them in anticlockwise order $a_{1}, r_{1}, b_{1}, t_{1}, c_{1}, u_{1}, d_{1}, v_{1}, \ldots, a_{g}, r_{g}, b_{g}, t_{g}, c_{g}$, $u_{g}, d_{g}, v_{g}, w_{1}, x_{1}, y_{1}, z_{1}, \ldots, w_{s-1}, x_{s-1}, y_{s-1}, z_{s-1}$. Let $F^{*}$ be the compact region bounded by all these geodesics, and choose the following isometries of the form $z \mapsto \frac{a z+\bar{c}}{c z+\bar{a}},|a|^{2}-|c|^{2}=1$. Let $S_{i}, i=1 \ldots, g$, be an isometry with $S_{i}\left(a_{i}\right)$ $=c_{i}, S_{i}\left(a_{i} \cap r_{i}\right)=c_{i} \cap t_{i}$, and $S_{i}\left(a_{i} \cap v_{i-1}\right)=c_{i} \cap u_{i}$. Let $T_{i}, i=1, \ldots, g$ be an isometry with $T_{i}\left(b_{i}\right)=d_{i}, T_{i}\left(b_{i} \cap r_{i}\right)=d_{i} \cap v_{i}$, and $T_{i}\left(b_{i} \cap t_{i}\right)=d_{i} \cap u_{i}$. Finally, for $i=1, \ldots, s-1$, let $U_{i}$ be an isometry with $U_{i}\left(w_{i}\right)=y_{i}, U_{i}\left(w_{i} \cap x_{i}\right)=y_{i} \cap x_{i}$, and $U_{i}\left(w_{i} \cap z_{i-1}\right)=y_{i} \cap z_{i}$ (using the notational conventions $v_{0}=z_{s-1}$ and $z_{0}=v_{g}$ ). See Figure 2. 
Let $G$ be the group generated by all the $S_{i}, T_{i}$ and $U_{i}$, and denote by $\Delta^{\prime}$ the image of $F^{*}$ under all the elements of $G$. Let $M^{\prime}=\Delta^{\prime} / G$ and $\pi: \Delta^{\prime} \rightarrow M^{\prime}$ be the natural projection. Then $M^{\prime}$ is a surface of genus $g$ with $s$ disks removed, and is thus diffeomorphic to $M$. The boundary components of $M^{\prime}$ are $\pi\left(x_{1}\right), \ldots, \pi\left(x_{s-1}\right)$ and $\pi\left(\bigcup_{i=1}^{g}\left(r_{i} \cup t_{i} \cup u_{i} \cup v_{i}\right) \bigcup_{i=1}^{s-1} z_{i}\right)$.

Proposition 12. In both the cases of zero and positive genus, there is a diffeomorphism $h: M^{\prime} \rightarrow M$ such that $h \circ \pi$ takes sides of the fundamental domain $F^{*}$ into minimal $\rho$-geodesic segments on $M$.

Proof. Denote by $\alpha_{1} \ldots \alpha_{2 g+s-1} \subset M^{\prime}$ the projections under $\pi$ of the sides of the fundamental domain $F^{*}$ in $\Delta^{\prime}$ which do not form boundary components. Choose some diffeomorphism $h^{\prime}: M \rightarrow M^{\prime}$ and denote by $\beta_{i} \in M^{\prime}$ a minimal $\rho$-geodesic in the free homotopy class corresponding to $\left[h^{\prime}\left(\alpha_{i}\right)\right]$. Note that the $\alpha_{i}$ are pairwise disjoint. We will first show that the $\beta_{i}$ are also pairwise disjoint.

Let $i \neq j$ and suppose that $x \in \beta_{i} \cap \beta_{j}$. We can lift $\beta_{i}$ and $\beta_{j}$ by $h^{\prime} \circ \pi$ to curve segments in $\Delta^{\prime}$ which intersect. These segments each have endpoints on sides of the fundamental domains which project to boundary components. Suppose they do not have endpoints on a common such side. Then it is clear that the lifts of $\beta_{i}$ and $\beta_{j}$ intersect at least twice. We can then construct a $\rho$-geodesic on $M$ with corners, by interchanging the arcs of $\beta_{i}$ and $\beta_{j}$ between two such intersections. This contradicts Lemma 10. Now suppose the lifts of $\beta_{i}$ and $\beta_{j}$ have endpoints on a common side. Then we may interchange the arcs between the common side and the point of intersection to again obtain a $\rho$-geodesic with corners, contradicting Lemma 10. Thus the $\beta_{i}$ are pairwise disjoint.

Now the construction of the diffeomorphism $h$ is clear. By cutting along the $\alpha_{i}$ we obtain a topological disk $D_{1}$. By cutting along the $\beta_{i}$ we obtain a different topological disk $D_{2}$. There is a homeomorphism $h^{\prime \prime}$ from $D_{1}$ to $D_{2}$ whose projection $h^{\prime \prime \prime}: M \rightarrow M^{\prime}$ after gluing takes $\alpha_{i}$ to $\beta_{i}$ for each $i$. Finally we smooth off the homeomorphism $h^{\prime \prime \prime}$ to obtain the required diffeomorphism $h$.

The metric $\rho$ lifts via $h \circ \pi$ to a metric $\widetilde{\rho}$ on $\Delta^{\prime}$.

\section{Properties of Class A $\widetilde{\rho}$-GeOdesics on SURFACES With Boundary}

In this section we define class $\mathrm{A}$ geodesics and show that there is a uniform constant bounding the distance a class A $\widetilde{\rho}$-geodesic segment in $\Delta^{\prime}$ can wander from the $h$-geodesic joining its endpoints. This is analogous to Lemma 8 of Morse [M1] and Lemma 7.1 of Hedlund [He]. We will also establish some other properties of class A $\widetilde{\rho}$-geodesic segments similar to those in [M1] and [He]. Finally we show that any class $\mathrm{A}$ geodesic that is not a boundary component of $\Delta^{\prime}$ is disjoint from $\partial \Delta^{\prime}$. We will use these facts in the next section to prove a theorem analogous to those of Morse and Hedlund, and hence deduce Theorem 2 in the case with boundary.

Definition 13. A $\widetilde{\rho}$-geodesic or $\widetilde{\rho}$-geodesic segment is of class $A$ if it minimises the distance between any two of its points in $\Delta^{\prime}$.

Lemma 14. Let $\alpha \neq \beta$ be class $A \widetilde{\rho}$-geodesic segments. Then the interiors of $\alpha$ and $\beta$ have at most one point of intersection. In particular, two distinct class $A$ $\widetilde{\rho}$-geodesics intersect at most once. 
Proof. (See [M1] Theorem 3.) Suppose $x \neq y$ are points of intersection of the interiors of $\alpha$ and $\beta$. Let the endpoints of $\alpha$ be $p$ and $q$ (labelled so that the points $p, x, y, q$ occur in order along $\alpha$ ). Let $\gamma_{1}$ be the segment of $\alpha$ from $p$ to $x, \gamma_{2}$ the segment of $\beta$ from $x$ to $y$ and $\gamma_{3}$ the segment of $\alpha$ from $y$ to $q$. Let $\gamma=\gamma_{1} \cup \gamma_{2} \cup \gamma_{3}$. Now, since $\alpha$ and $\beta$ are class A, we have $L_{\tilde{\rho}}(\alpha ; x, y)=L_{\tilde{\rho}}(\beta ; x, y)$ and so $L_{\tilde{\rho}}(\alpha ; p, q)=L_{\tilde{\rho}}(\gamma ; p, q)$. Hence $\gamma$ is also a class A $\tilde{\rho}$-geodesic segment. But since $\alpha \neq \beta, \gamma$ has corners at $x$ and $y$, contradicting Lemma 10.

The following lemma will be used to establish the uniform bound of the distance between $h$-geodesic segments and class A $\widetilde{\rho}$-geodesic segments with the same endpoints.

Lemma 15. Let $L, \alpha:[0,1] \rightarrow \Delta^{\prime}$ have the same endpoints, where $L$ is an $h$ geodesic segment and $\alpha$ is a class $A \tilde{\rho}$-geodesic segment. Let $F_{1}, \ldots, F_{m}$ be the copies of the fundamental domain $F^{*}$ through which $L$ passes, in order. Then the fundamental domains through which $\alpha$ passes are also, in order, $F_{1}, \ldots, F_{m}$.

Proof. First note that neither $L$ nor $\alpha$ crosses the same side of a fundamental domain more than once. This is true for $L$, as distinct $h$-geodesics intersect at most once. It is true for $\alpha$ by Lemma 14. Since bounding geodesics of the fundamental domains are disjoint, it is clear that if $\alpha$ crosses a side $a$ of a fundamental domain that $L$ does not cross, it enters the half plane bounded by $a$ not containing points of $L$ and must thus cross $a$ again. Hence the lemma is proved.

Since $M$ is compact, we may define

$$
d=\sup _{x, y \in F^{*}} d_{h}(x, y)<\infty
$$

for our fundamental domain $F^{*}$. Now we have

Proposition 16. Let $\gamma$ be a class $A \tilde{\rho}$-geodesic segment in $\Delta^{\prime}$ and let $L$ be the h-geodesic segment joining its endpoints. Then

$$
\sup _{y \in L} \inf _{x \in \gamma} d_{h}(x, y) \leq d
$$

and

$$
\sup _{x \in \gamma} \inf _{y \in L} d_{h}(x, y) \leq d .
$$

Proof. Let $x \in \gamma$, and $F$ be the copy of $F^{*}$ containing $x$. Then by Lemma 15 , $L \cap F \neq \emptyset$, so $\inf _{y \in L} d_{h}(x, y) \leq d$, hence $\sup _{x \in \gamma} \inf _{y \in L} d_{h}(x, y) \leq d$. Similarly, $\sup _{y \in L} \inf _{x \in \gamma} d_{h}(x, y) \leq d$.

Lemma 17. The set of class A $\tilde{\rho}$-geodesics is closed in the set of all $\widetilde{\rho}$-geodesics.

Proof. (See [M1] Lemma 9.) We need to show that if $\gamma_{n}$ are class A $\tilde{\rho}$-geodesics and $\gamma_{n} \rightarrow \gamma$ then $\gamma$ is also class A. Suppose not. Then there are points $p, q \in \gamma$ and a geodesic segment $\delta$ with endpoints $p, q$ such that $L_{\tilde{\rho}}(\delta)<L_{\tilde{\rho}}(\widehat{\gamma})$ where $\widehat{\gamma}$ is the segment of $\gamma$ with endpoints $p, q$. Let $\epsilon=L_{\tilde{\rho}}(\widehat{\gamma})-L_{\tilde{\rho}}(\delta)$. Now, since $\gamma=\lim \gamma_{n}$, there is a $\gamma_{n}$ containing a segment $\widehat{\gamma}_{n}$ whose endpoints $r, s$ satisfy $d_{\tilde{\rho}}(r, p)<\epsilon / 4$, $d_{\tilde{\rho}}(s, q)<\epsilon / 4$ and such that $\left|L_{\tilde{\rho}}\left(\widehat{\gamma}_{n}\right)-L_{\tilde{\rho}}(\widehat{\gamma})\right|<\epsilon / 2$. But then there is a curve $\alpha$ 
with endpoints $r, s$ such that

$$
\begin{aligned}
L_{\tilde{\rho}}(\alpha) & <\epsilon / 4+\epsilon / 4+L_{\tilde{\rho}}(\delta) \\
& <L_{\tilde{\rho}}(\widehat{\gamma})-\epsilon / 2 \\
& <L_{\tilde{\rho}}\left(\widehat{\gamma}_{n}\right),
\end{aligned}
$$

contradicting the fact that $\gamma_{n}$ is class A.

Lemma 18. Let $\gamma$ be a $\widetilde{\rho}$-geodesic or $\widetilde{\rho}$-geodesic segment which is not a boundary component of $\Delta^{\prime}$. Then $\gamma$ does not intersect the boundary of $\Delta^{\prime}$, except possibly at an endpoint.

Proof. Suppose otherwise. Then by Lemma 10, $\gamma$ would meet a boundary component of $\Delta^{\prime}$ tangentially. But this contradicts Lemma 9.

\section{Proof of Theorem 2 For Surfaces With boundary}

Now we have established Proposition 16, we proceed to prove a theorem analogous to Morse's [M1]. Then we deduce Theorem 2 in the case with boundary.

We remark that by Lemma 18, we may assume all geodesics in which we are interested lie in the interior of $\Delta^{\prime}$.

Two curves $\alpha, \beta \subset \Delta$ are said to be of the same type if there exists a constant $R$ such that for each $x \in \alpha$ there exists $y \in \beta$ with $d_{h}(x, y) \leq R$ and for each $y \in \beta$ there exists $x \in \alpha$ with $d_{h}(y, x) \leq R$.

Theorem 19. Given any h-geodesic in $\Delta^{\prime}$ there is a class $A \widetilde{\rho}$-geodesic of the same type. Conversely, given any class $A \tilde{\rho}$-geodesic which extends infinitely in both directions, there is a unique h-geodesic of the same type.

Proof. Let $L$ be an $h$-geodesic in $\Delta^{\prime}$. Let $\ldots, P_{-2}, P_{-1}, P_{0}, P_{1}, P_{2}, \ldots$ be successive points on $L$ with $d_{h}\left(P_{n}, P_{0}\right) \rightarrow \infty$ as $|n| \rightarrow \infty$. Choose a class A $\widetilde{\rho}$-geodesic segment $\gamma_{n}$ with endpoints $P_{-n}$ and $P_{n}$. By Lemma 16 there is a point $Q_{n} \in \gamma_{n}$ with $d_{h}\left(Q_{n}, P_{0}\right) \leq d$. Let $x_{n} \in S^{\tilde{\rho}} \Delta^{\prime}$ be the unit tangent vector determined by $Q_{n}$ and the geodesic $\gamma_{n}$. All the $x_{n}$ lie in the restriction of $S^{\tilde{\rho}} \Delta^{\prime}$ to $\left\{z \in \Delta^{\prime}: d_{h}\left(z, P_{0}\right) \leq d\right\}$, which is compact; hence they have a limit point $x$. Let $\gamma$ be the geodesic determined by $x$. Now $\gamma$ is class A by Lemma 17 , and

$$
\sup _{z \in \gamma} \inf _{y \in L} d_{h}(z, y) \leq d
$$

by Lemma 16 , so $\gamma$ has the same type as $L$.

To prove the converse, let $\gamma$ be a class A geodesic extending infinitely in both directions. Let $\ldots, P_{-1}, P_{0}, P_{1}, \ldots$ be successive points on $\gamma$ with $d_{h}\left(P_{n}, P_{0}\right) \rightarrow \infty$ as $|n| \rightarrow \infty$. Let $L_{n}$ be the $h$-geodesic segment with endpoints $P_{-n}, P_{n}$. By Lemma 16 there is a point $Q_{n} \in L_{n}$ with $d_{h}\left(Q_{n}, P_{0}\right) \leq d$. As before, define $x_{n} \in S^{h} \Delta^{\prime}$ by $Q_{n}$ and the $h$-geodesic $L_{n}$ and let $x$ be a limit point of the $x_{n}$. Let $L$ be the $h$-geodesic defined by $x$. Again $L$ is of the same type as $\gamma$ since all the $L_{n}$ are. Since $L$ is of the same type as $\gamma$ and $\gamma \in \Delta^{\prime}$, we must have $L \in \Delta^{\prime}$. To prove uniqueness, note that any two $h$-geodesics of the same type are equal.

We now show how to construct the semi-equivalence $(\Theta, \tau)$ of our Theorem 2, between the set $\mathcal{A}$ and the set $Z^{h}$. Recall that $\mathcal{A} \subset S^{\rho} M$ is the set of unit tangent vectors which correspond to projections of class A $\widetilde{\rho}$-geodesics. Let $\widetilde{\mathcal{A}} \subset S^{\tilde{\rho}} \Delta^{\prime}$ be the set of unit tangent vectors to $\Delta^{\prime}$ which correspond to class A $\widetilde{\rho}$-geodesics. Denote by $\lambda$ the natural projection $\lambda: S^{\tilde{\rho}} \Delta^{\prime} \rightarrow \Delta^{\prime}$ and by $\mu$ the natural projection 
$\mu: S^{h} \Delta^{\prime} \rightarrow \Delta^{\prime}$. The boundary of our fundamental domain $F^{*}$ (we shall drop the star from now on) is formed from a finite number of simultaneously class A $\widetilde{\rho}$-geodesic segments and $h$-geodesic segments. Let $x \in \widetilde{\mathcal{A}}$ with $\lambda(x) \in \partial F$ and suppose that $\lambda\left(\phi_{t}^{\tilde{\rho}}(x)\right) \in F$ for $t$ small and positive (that is, $x$ represents a vector on the boundary of $F$ pointing into $F$, and corresponding to a class A $\widetilde{\rho}$-geodesic). Let $y \in \widetilde{\mathcal{A}}$ have $\lambda(y) \in \partial F, \phi_{t}^{\tilde{\rho}}(y) \notin F$ for $t>0$ and $y$ in the forward orbit of $x$ under $\phi^{\tilde{\rho}}$ (that is, $y$ represents the vector above the boundary of $F$ pointing out of $F$, and along the same geodesic as $x$ ). Let $\gamma$ be the class A $\widetilde{\rho}$-geodesic defined by $x$ and $y$, and let $L$ be the $h$-geodesic of the same type as $\gamma$. Let $p, q \in S^{h} \Delta^{\prime}$ satisfy $\mu(p), \mu(q) \in \partial F \cap L, \mu\left(\phi_{t}^{h}(p)\right), \mu\left(\phi_{-t}^{h}(q)\right) \in F$ for $t$ small and positive (that is, $p$ and $q$ are the entry and exit vectors of $L$ in the fundamental domain $F$ ). Define $\theta(x)=p, \theta(y)=q$ and define $\theta$ on points of the flow from $x$ to $y$ by linear interpolation along the geodesic segment, that is, suppose $z \in \widetilde{\mathcal{A}}$ with $\lambda(z) \in F \cap \gamma$, and $z$ in the orbit of $x$ and $y$. There is $r \in S^{h} \Delta^{\prime}$ with $\mu(r) \in L \cap F, r$ in the orbit of $p$ and $q$, and

$$
\frac{d_{h}(\mu(p), \mu(r))}{d_{h}(\mu(p), \mu(q))}=\frac{d_{\tilde{\rho}}(\lambda(x), \lambda(z))}{d_{\tilde{\rho}}(\lambda(x), \lambda(y))} .
$$

Then let $\theta(z)=r$. We extend the definition of $\theta$ to the whole of $\widetilde{\mathcal{A}}$ by translation by the group elements $g \in G$.

The linear interpolation induces a reparametrization $\tau: \widetilde{\mathcal{A}} \times \mathbb{R} \rightarrow \mathbb{R}$, by

$$
\begin{gathered}
\tau(z, 0)=0 \text { for all } z \in \widetilde{\mathcal{A}}, \\
\frac{d \tau}{d t}=\frac{d_{\tilde{\rho}}(\lambda(x), \lambda(y))}{d_{h}(\mu(p), \mu(q))}
\end{gathered}
$$

and we extend the definition of $\tau$ to the whole of $\widetilde{\mathcal{A}} \times \mathbb{R}$ by translation by the group elements $g \in G$ and addition of the changes in $\tau$ across each copy $g F$ of the fundamental domain. Now we have:

Proposition 20. $\theta: \widetilde{\mathcal{A}} \rightarrow \widetilde{Z^{h}}$ is a continuous surjection and induces a map $\Theta$ : $\mathcal{A} \rightarrow Z^{h} . \tau$ is continuous and

$$
\phi^{h} \circ \Theta=\left.\Theta \circ \phi_{\tau}^{\rho}\right|_{\mathcal{A} \times \mathbb{R}} .
$$

Proof. Surjectivity of $\theta$ follows from Theorem 19. We will show continuity. Let $x_{n} \in \widetilde{\mathcal{A}}$ and suppose $x_{n} \rightarrow x$. Then $x \in \widetilde{\mathcal{A}}$ by Lemma 17. Let $\gamma_{n}$ be the class A $\widetilde{\rho}$-geodesic corresponding to $x_{n}, \gamma$ the class $\mathrm{A} \widetilde{\rho}$-geodesic corresponding to $x, L_{n}$ the $h$-geodesic of the same type as $\gamma_{n}$ and $L$ the $h$-geodesic of the same type as $\gamma$. For each curve $\alpha$ denote by $\widehat{\alpha}$ the segment $\alpha \cap F$. It is clear that the $\widehat{L}_{n}$ accumulate on $\widehat{L}$, the $\widehat{\gamma}_{n}$ on $\widehat{\gamma}$ and that the lengths of the segments converge: $L_{\tilde{\rho}}\left(\widetilde{\gamma}_{n}\right) \rightarrow L_{\tilde{\rho}}(\widehat{\gamma})$ and $L_{h}\left(\widehat{L}_{n}\right) \rightarrow L_{h}(\widehat{L})$. Thus we have

$$
\lim _{n \rightarrow \infty} \theta\left(x_{n}\right)=\theta(x)
$$

for $x$ in the interior of any fundamental domain. To see continuity of $\theta$ across the boundaries of the fundamental domains, it is enough to observe that for $g \in G, \gamma$ is of the same type as $L$ if and only if $g \gamma$ is of the same type as $g L$. The same argument gives continuity of $\tau(z, t)$ with respect to $z$. Continuity of $\tau$ with respect to $t$ is immediate. 
That $\theta$ projects to a well defined map $\Theta: \mathcal{A} \rightarrow Z^{h}$ follows from the facts that, for any $g \in G$, the metrics $\widetilde{\rho}$ and $h$ are invariant under $g$ and the class A $\widetilde{\rho}$-geodesic $\gamma$ is of the same type as the $h$-geodesic $L$ if and only if $g \gamma$ is of the same type as $g L$. The final statement of the proposition is immediate.

Note that in general $\Theta$ does not descend from the unit tangent bundle to the surface, because the 'orientation' in which three class A geodesics cross need not be the same as for their hyperbolic shadows.

\section{Proof of Theorem 2 in Case Without boundary}

In this section we adapt our method of proof to the case without boundary, obtaining a new proof of the main Lemma of Morse's paper ([M1] Lemma 8), and sketching how this leads to Theorem 2 in this case.

Let $M$ be a compact surface of genus $g \geq 2$ without boundary. We use Koebe's construction [BS] which defines a universal cover with useful properties, namely we cut the surface along curves in the free homotopy classes indicated in Figure 3.

We choose the curves to be minimal $\rho$-geodesics in their free homotopy class by Lemma 8 . This implies, by Lemma 14, that each curve intersects each other curve at most once. Hence, by the choice of free homotopy classes, the numbers of intersections are precisely as indicated in Figure 3. The covering space, one fundamental domain, and generators for the group of deck transformations are shown in Figure 4 (for the case $g=2$ ); each fundamental domain is a $(8 g-4)$-gon.

Let $G$ be the group of transformations with $\Delta / G \cong M$. Since each cutting curve on $M$ is a periodic curve intersecting at most two other cutting curves, each $h$-geodesic containing a side of a fundamental domain is a $\widetilde{\rho}$-geodesic intersecting only its two immediate neighbours. These curves are periodic, that is, for each such geodesic $a$ there is $g \in G$ with $g a=a$. First we show that these are class A $\tilde{\rho}$-geodesics.

Lemma 21. Let $\gamma$ be a $\widetilde{\rho}$-geodesic of period $g^{k}$ which projects onto the shortest closed $\rho$-geodesic in its homotopy class. Then $\gamma$ has period $g$.

Proof. First observe that $\gamma$ minimises the distance between any point $p \in \gamma$ and $g^{k} p$; also that $\gamma$ has endpoints on $S^{1}$ which are fixed points of $g$.

Suppose $g p \notin \gamma$. We will call the half plane bounded by $\gamma$ which contains points on the right of $\gamma$ when proceeding from some point $q$ to $g^{k} q$ the positive side of $\gamma$, the other half plane bounded by $\gamma$ will be the negative side of $\gamma$. Suppose $g p$ lies in the positive side of $\gamma$ (the case for the negative side is identical). Consider now the $\tilde{\rho}$-geodesics $g^{i} \gamma$ for $i=0, \ldots, k-1$. For each $i$, the point $g^{i+1} p$ lies on the positive side of $g^{i} \gamma$. It follows that some geodesic $g^{j} \gamma$ intersects $\gamma$ twice between two points $q, g^{k} q \in \gamma$. This contradicts the minimality of $\gamma$ and $g^{j} \gamma$. Hence $g^{i} p \in \gamma$ for each $i$. Now we have that $p, g p \in \gamma \cap g \gamma$, so by the same argument $\gamma=g \gamma$.

Lemma 22. Let $\gamma$ be a $\widetilde{\rho}$-geodesic of period $g \in G$ which projects onto the shortest closed $\rho$-geodesic in the appropriate homotopy class. Then $\gamma$ is a class A $\widetilde{\rho}$-geodesic.

Proof. Suppose not. Then there are points $p, q \in \gamma$ and a curve $\alpha$ such that $L_{\tilde{\rho}}(\alpha ; p, q)<L_{\tilde{\rho}}(\gamma ; p, q)$. Let $k$ be the smallest positive integer such that there is $\bar{p} \in \gamma$ with $p, q$ lying in the interior of the segment of $\gamma$ with endpoints $\bar{p}$ and $g^{k} \bar{p}$. By minimality of the curve, $k>1$. Now let $L=L_{\tilde{\rho}}(\gamma ; \bar{p}, g \bar{p})$. Then $L_{\tilde{\rho}}\left(\gamma ; \bar{p}, g^{k} \bar{p}\right)=k L$. Now the curve consisting of the segment of $\gamma$ from $\bar{p}$ to $p$, the segment of $\alpha$ from 


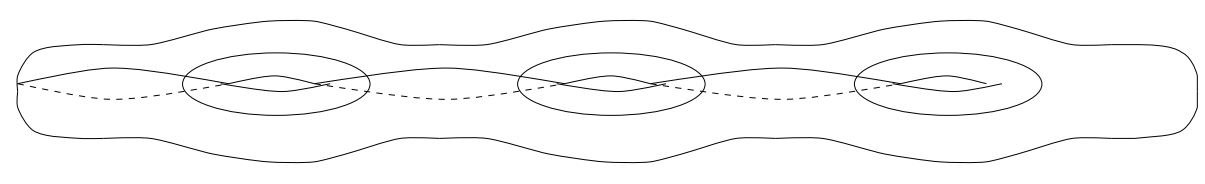

Figure 3. (for the case $g=3$ ).

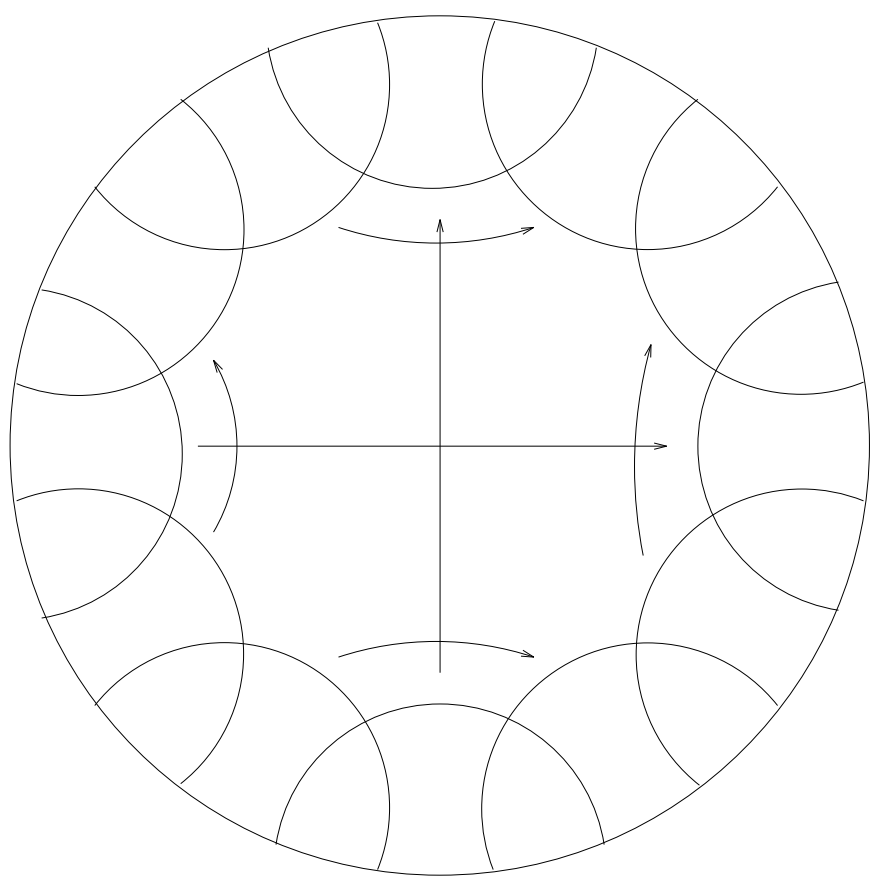

Figure 4. (for the case $g=2$ ).

$p$ to $q$, and the segment of $\gamma$ from $q$ to $g^{k} \bar{p}$, has length less than $k L$. Now let $\beta$ be the shortest curve in $\Delta$ joining points $r$ and $g^{k} r$ for some $r$. Then $\beta$ has length less than $k L$. Clearly the curve consisting of $\beta$ and all its images under $g^{n}, n \in \mathbb{Z}$, is periodic and is a geodesic (if it contains corners at $g^{n} r$, then there is a shorter curve with the given properties). But by Lemma 21, the curve so generated by $\beta$ has period $g$, and so there is a curve of period $g$ which projects onto a curve on $M$ of length less than that of the projection of $\gamma$, contradicting the minimality of $\gamma$.

Hence we see that each class A $\widetilde{\rho}$-geodesic different from a boundary geodesic of the fundamental domains intersects any given boundary geodesic of the fundamental domains at most once.

The next lemma is analogous to Lemma 15 , but slightly weaker.

Lemma 23. Let $\gamma$ be a class $A \tilde{\rho}$-geodesic segment and let $L$ be the h-geodesic with the same endpoints. Let $L$ pass through a fundamental domain $F$. Then $\gamma$ either passes through $F$ or passes through a fundamental domain which touches $F$ at a vertex. Conversely, let $\gamma$ pass through a fundamental domain $F^{\prime}$. Then $L$ either 
$\mathrm{F}$

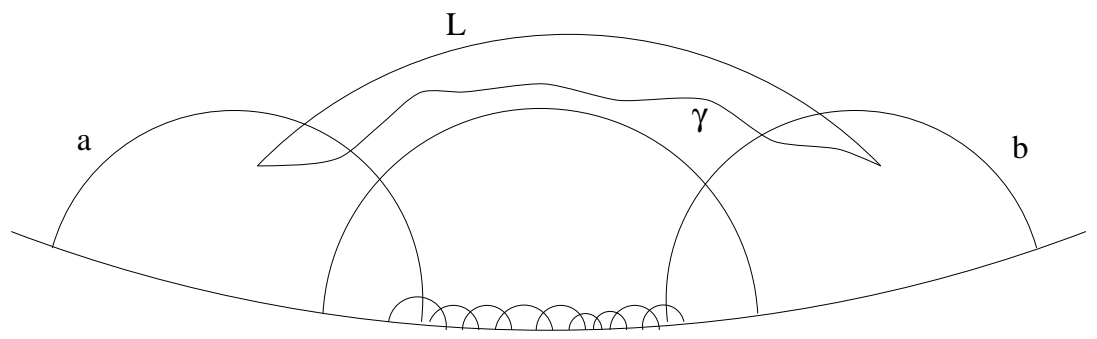

Figure 5

F

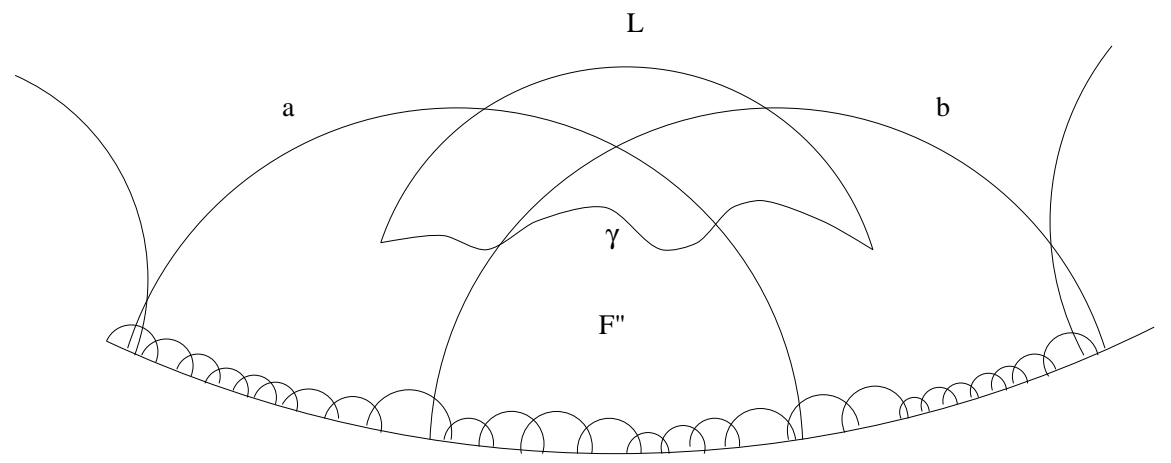

FIGURE 6

passes through $F^{\prime}$ or passes through a fundamental domain which touches $F^{\prime}$ at a vertex.

Proof. Suppose $L$ passes through $F$. If either endpoint of $L$ is in $F$, then we are done. Suppose then that no endpoints of $L$ are in $F$.

Suppose firstly that $L$ intersects two sides $a, b$ of $F$ which are not adjacent. Then $\gamma$ must also intersect the $h$-geodesics containing each of these sides. Since these $h$ geodesics are disjoint, if $\gamma \cap F=\emptyset$ then $\gamma$ must intersect a geodesic containing a side of $F$ lying between $a$ and $b$ at least twice. See Figure 5 . We have just shown this is impossible, so $\gamma \cap F \neq \emptyset$.

Now suppose $L$ intersects two adjacent sides $a, b$ of $F$. Denote by $F^{\prime \prime}$ the fundamental domain which meets $F$ only at the vertex of $a$ and $b$. Again, $\gamma$ must intersect the $h$-geodesics containing $a$ and $b$. Suppose $\gamma$ does so in the same order as $L$, then clearly $\gamma \cap F \neq \emptyset$. Suppose $\gamma$ intersects $a$ and $b$ in the opposite order to $L$, then we have $\gamma \cap F^{\prime \prime} \neq \emptyset$. See Figure 6 .

The proof of the converse is similar. The only property we have used of the geodesic $\gamma$ is that it does not intersect any boundary arc of a fundamental domain more than once. Since the boundary arcs of fundamental domains are $h$-geodesics the same is true for $L$, so the rôles of $\gamma$ and $L$ in this lemma are interchangeable.

Lemma 24 (See [M1] Lemma 8). Let

$$
d=\sup _{x, y \in F} d_{h}(x, y)
$$


for our fundamental domain $F$. Let $\gamma, L$ be as in Lemma 23. Then

$$
\sup _{x \in \gamma} \inf _{y \in L} d_{h}(x, y) \leq 2 d
$$

and

$$
\sup _{y \in L} \inf _{x \in \gamma} d_{h}(x, y) \leq 2 d .
$$

Proof. This is immediate from Lemma 23.

Having established Lemma 24, we may proceed in the same manner as Morse [M1] (or the proof of Theorem 19) to prove

Theorem 25 ([M1]). Given any h-geodesic $l$ in $\Delta$ there is a class $A \widetilde{\rho}$-geodesic $\alpha$ of the same type as l. Conversely, given any class $A \widetilde{\rho}$-geodesic there is a unique h-geodesic of the same type.

To establish the semi-equivalence $\theta$ similar to that in Proposition 20, we need to exercise a little more care. Specifically, to ensure that $\theta$ is well defined, we use a linear interpolation along class A geodesics $\gamma$ and the hyperbolic geodesics $L$ of the same type as $\gamma$ between entry points of fundamental domains $F$ and $F^{\prime}$ where $\gamma \cap F, L \cap F, \gamma \cap F^{\prime}$ and $L \cap F^{\prime}$ are all non-empty.

As before, let $\widetilde{\mathcal{A}} \subset S^{\tilde{\rho}} \Delta$ be the set of unit tangent vectors corresponding to class A $\tilde{\rho}$-geodesics.

Proposition 26. There is a semi-equivalence $(\theta, \tau)$ from $\left(\phi^{\widetilde{\rho}}, \widetilde{\mathcal{A}}\right)$ to $\left(\phi^{h}, S^{h} \Delta\right)$ which induces a semi-equivalence $(\Theta, \tau)$ from $\left(\phi^{\rho}, \mathcal{A}\right)$ to $\left(\phi^{h}, S^{h} M\right)$.

Proof. We outline the proof, showing only those parts which are different from the proof of Proposition 20.

Fix some fundamental domain $F$. Suppose $x \in \mathcal{A}$ satisfies:

(i) $\lambda(x) \in \partial F$,

(ii) $\lambda\left(\phi_{t}^{\tilde{\rho}}(x)\right) \in F$ for $t$ small and positive, and

(iii) if $x$ corresponds to a class A $\widetilde{\rho}$-geodesic $\gamma$ and $L$ is the $h$-geodesic of the same type as $\gamma$, then $L \cap F \neq \emptyset$.

Then let $r \in S^{h} \Delta$ be the unit tangent vector corresponding to the $h$-geodesic $L$ satisfying $\mu(r) \in \partial F$ and $\mu\left(\phi_{t}^{h}(r)\right) \in F$ for $t$ small and positive, and define $\theta(x)=r$. Now suppose $y \in \mathcal{A}$ is in the forward orbit of $x$ and is the first such vector satisfying $\lambda(y) \in \partial F^{\prime}$ for some fundamental domain $F^{\prime} \neq F$ with $F^{\prime} \cap L \neq \emptyset$. Let $s$ be the first vector in the forward orbit of $r$ with $\mu(s) \in \partial F^{\prime}$. Then define $\theta(y)=s$, and use linear interpolation along $\gamma$ from $x$ to $y$ to define $\theta$ on vectors in $\mathcal{A}$ representing the segment of $\gamma$ from $x$ to $y$. We extend the definition of $\theta$ to points in $\widetilde{\mathcal{A}}$ above other fundamental domains by translation by group elements.

It is clear from the proof of Lemma 23 that if $\gamma$ passes from the fundamental domain $F_{1}$ into the fundamental domain $F_{2}$ then $L \cap\left(F_{1} \cup F_{2}\right) \neq \emptyset$. This ensures that $\theta$ is defined on all of $\widetilde{\mathcal{A}}$. Condition (iii) above ensures that we define $\theta$ on each $z \in \widetilde{\mathcal{A}}$ only once, that is, $\theta$ is well-defined.

This defines a continuous surjection from $\mathcal{A}$ to $S^{h} \Delta$ which, after the corresponding time-reparametrization, commutes with the flow, hence a semiequivalence between the flow generated by class A $\rho$-geodesics and that generated by $h$-geodesics on the surface $M$. 
Propositions 20 and 26 between them imply Theorem 2 in the case of a surface without boundary.

\section{Comments}

We conclude with a number of comments on relations with other work and directions of potential development.

Remark 27. A sample of connections with other work. Hedlund [He] proved an analogous result to Morse's for surfaces of genus 1, where the reference metric is flat instead of constant negative curvature. Theorem $5 \cdot 3 \cdot 6$ of $[\mathrm{K}]$ is analogous to the main Theorem of [M1] for manifolds of arbitrary dimension, but has weaker conclusion than our main result. In a later paper [M2], Morse showed that with certain conditions on the metric $\rho$ ('uniform instability'), the semi-equivalence is an equivalence (i.e. $\Theta$ is a homeomorphism) and in this case $\mathcal{A}$ is the whole of $S^{\rho} M$. This idea was greatly developed by Anosov and others (see [Pe] for a survey). More recently, both Polterovich $[\mathrm{Po}]$ and Bangert [Ba] proved that results similar to Morse's [M1] hold for a 'torus with a big bump'. The results presented in the present paper were motivated by a desire to replace the metric property above (the 'big bump') with topological properties. Related results were obtained by Bolotin [Bol1], [Bol2]. The periodic orbits we find could also be obtained by a method of Angenent using the curvature flow on closed curves; in fact his method would give not just class A geodesics but many of minimax and other types. The idea of global shadowing was taken up by Katok (e.g. see Handel [Ha]). It also links with Gromov's quasi-geodesics, which merit a remark of their own.

Remark 28. Gromov's quasi-geodesics. Thierry Barbot pointed out to us that our result could be obtained as a corollary of Gromov's theory of quasi-geodesics $[\mathrm{Gr}],[\mathrm{CDP}],[\mathrm{GH}]$. This approach has the added advantage that it generalises easily to arbitrary dimension (see Remark 31 below). A Riemannian manifold is Gromov-hyperbolic if the universal cover has the thin triangle property, i.e. there is a $\delta$ such that for every triangle formed from class A geodesics, each side is within $\delta$ of the union of the other two. For a compact manifold this is a property of the fundamental group only. In particular it holds for all compact surfaces of negative Euler characteristic. Given a metric $h$ (e.g. a hyperbolic one), a parametrised curve $x(t)$ is called a $(C, D)$-quasi-geodesic if for all $t, t^{\prime}, C^{-1}\left|t^{\prime}-t\right|-D \leq d_{h}\left(x(t), x\left(t^{\prime}\right)\right) \leq$ $C\left|t^{\prime}-t\right|+D$ on the universal cover. In particular, given any other metric $g$ there exist $C, D$ such that class A geodesics of $g$ are $(C, D)$-quasi-geodesics with respect to $h$. Gromov's theory shows that there exists $R$ depending only on $C, D$ such that every quasi-geodesic has a class A $h$-geodesic within $R$ of it. The same result can be applied in the other direction. Then existence of the semiconjugacy follows fairly easily. The approach of our paper does have two advantages, nonetheless. The first is that it gives an explicit and tight bound on the distance a class A geodesic can wander from a hyperbolic geodesic (Lemma 15 for the case with boundary and Lemma 23 for the case without). Secondly, it constructs a periodic class A geodesic representing each nontrivial free homotopy class.

Remark 29. Mechanical systems. Our theorem applies in principle to some mechanical systems on surfaces $M$, with Lagrangian of the form $L=T(\dot{q})-V(q)$, where $T(\dot{q})=\frac{1}{2}|\dot{q}|^{2}$ is defined by a Riemannian metric on $M$. Given $E \in \mathbb{R}$, the 
dynamics on the energy surface where $T+V=E$ are equivalent to those of the geodesic flow of the Jacobi-Maupertuis metric $d s^{2}=(E-V(q))|d q|^{2}$ (e.g. [Ar]). If $E>V_{\max }=\max \{V(q): q \in M\}$ and $M$ is a closed surface of genus at least 2 then we can apply Morse's Theorem and deduce complicated dynamics. If $E<V_{\max }$ then the Jacobi-Maupertuis metric is degenerate on the boundary of $M_{E}=\{q \in M: V(q)<E\}$, but we can still apply our theorem if each boundary component of $M_{E}$ is surrounded by a closed geodesic and the surface remaining after cutting round these geodesics has negative Euler characteristic. For example, our theorem can be applied to Bolotin's examples [Bol1], [Bol2], leading to stronger conclusions than he obtained. One might have hoped that the boundary components of $M_{E}$ could be modelled by cusps (then we could adapt our theorem to allow punctures and deduce similar results), but this is not the case in general, as the distance to the boundary is finite.

Remark 30. Fermi surfaces. The Fermi surface of a 3D crystal is a surface (or union of surfaces) in the reciprocal space of the lattice, describing the allowed wavemomenta of the conduction electrons (e.g. $[\mathrm{AM}]$ ). It often has genus greater than one. For example, for copper, silver and gold it has genus 4. There is a natural metric on the Fermi surface, called the inverse mass matrix. We do not yet know of a physical interpretation of its geodesics, but can already predict that the geodesic flow is complicated.

Remark 31. Higher-dimensional analogues. Our method will not generalize to higher dimensions since it uses the fact that a geodesic in the Poincaré disk separates the disk. But by Remark 28, an analogous result does hold for compact manifolds of any dimension provided the fundamental group is Gromov-hyperbolic (i.e. its Cayley graph has the thin triangle property). This idea has recently been developed by Golé and Boyland in the context of time-dependent Lagrangian systems.

Remark 32. Indefinite metrics. It would be very interesting to try to extend these ideas to the geodesic flow of indefinite metrics (e.g. Minkowski). A recent paper addressing related issues is $[\mathrm{Bu}]$.

\section{ACKNOWLEDGEMENTS}

We are grateful to Phil Boyland, John Parker, Caroline Series and Thierry Barbot for useful comments. The completion of the work benefitted from the hospitality of the Laboratoire de Topologie, Université de Bourgogne, France, and membership in an EC network on Stability and Universality in Classical Mechanics and an INTAS collaboration on Hamiltonian dynamics and bifurcations.

\section{REFERENCES}

[Ar] Arnol'd, V.I., Mathematical methods of classical mechanics, Springer Verlag, (1978). MR $\mathbf{5 7 : 1 4 0 3 3 b}$

[AM] Ashcroft, N.W. and Mermin, N.D., Solid State Physics, W. B. Saunders, (1976).

[Ba] Bangert, V., Mather Sets for Twist Maps and Geodesics on Tori, Dynamics Reported, 1, (U. Kirchgraber and H.O. Walther, eds), Wiley, (1988), pp 1-56. MR 90a:58145

[Be] Beardon, A.F., The Geometry of Discrete Groups, Springer-Verlag, (1982). MR 85d:22026

[Bi] Birkhoff, G.D., Dynamical Systems with Two Degrees of Freedom, Trans. AMS, 18, (1917), 219. 
[BS] Birman, J. and Series, C., Dehn's Algorithm Revisited, with Applications to Simple Closed Curves on Surfaces, Proc. Conf. on Combinatorial Group Theory, Utah (Gerston and Stallings, eds.), (1984), Princeton Univ. Press, pp 451-478. MR 88k:20059

[Bol1] Bolotin, S., Homoclinic orbits of geodesic flows on surfaces, Russian J Math Phys 1 (1993) 275-283. MR 94k:58107

[Bol2] Bolotin, S., Variational criteria for nonintegrability and chaos in Hamiltonian systems, Proc NATO ASI, Torun, 1993, ed. Seimenis J. MR 95m:58054

[Boy] Boyland, P.L., Topological methods in surface dynamics, Topology and its Applications 58, (1994), 223-298. MR 95h:57016

[Bu] Buffoni, B., Periodic and homoclinic orbits for Lorentz-Lagrangian systems via variational methods, Nonlinear Analysis, 26 (1995), 443-462. MR 97a:58028

[C] Carathéodory, C., Calculus of Variations and Partial Differential Equations of the First Order. Part II: Calculus of Variations, Holden-Day. (1967). MR 38:590

[CDP] Coornaert, M., Delzant, T., Papadopoulos, A., Géométrie et théorie des groupes, les groupes hyperboliques de Gromov, Lecture Notes in Math 1441, (Springer), (1990). MR 92f: 57003

[Gr] Gromov, M., Hyperbolic Groups, Essays in group theory (Gersten S.M., ed.), (Springer), (1987), 75-263. MR 89e:20070

[GH] Guivarc'h, Y., de la Harpe, P. (eds), Sur les groupes hyperboliques d'après M.Gromov, Progress in Math 83, (Birkhäuser), (1990). MR 92f:53050

[Ha] Handel, M., Global shadowing of pseudo-Anosov homeomorphisms, Ergod Th Dyn Sys 5, (1985), 373-377. MR 87e:58172

[He] Hedlund, G.A., Geodesics on a Two-Dimensional Riemannian Manifold with Periodic Coefficients, Ann. Math., 33 (1932) 719-739.

[K] Klingenberg, W., Lectures on Closed Geodesics, Springer-Verlag. (1978). MR 57:17563

[LY] Li, T-Y., and Yorke, J.A., Period three implies chaos, Amer. Math. Monthly, 82 (1975) 985-992.

[M1] Morse, H.M., A Fundamental Class of Geodesics on any Closed Surface of Genus Greater than One, Trans. AMS, 26, (1924), 25-60.

[M2] Morse, H.M., Instability and Transitivity, Journ. de Math, XIV, (1935), 49-71.

[Pe] Pesin, Ya. B., General theory of smooth hyperbolic dynamical systems, in Dynamical Systems vol II, (Sinai Ya. G. ed.), (Springer), (1989). MR 91i:58079

[Po] Polterovich, L.V., Geodesics on the Two-Dimensional Torus with Two Rotation Numbers, Math. USSR Izvestiya, 33, 1. (1989), 101-114. MR 89m:58166

[S1] Series, C., Geometrical Markov Coding of Geodesics on Surfaces of Constant Negative Curvature, Ergodic Theory and Dynamical Systems, 6, (1986), 601-625. MR 88k:58130

[S2] Series, C., Some Geometrical Models of Chaotic Dynamics, Proc. R. Soc. London A, 413, (1987),171-182. MR 89g:58169

[Sh] Sharkovskii, AN, Co-existence of the cycles of a continuous mapping of the line into itself, Ukrain Math Zh 16:1 (1964) 61-71 (Russian).

Mathematics Institute, University of Warwick, Coventry CV4 7AL, UK

Current address, J. Denvir: Department of Mathematics, Truman State University, Kirksville, Missouri 63501

E-mail address: jdenvir@math.truman.edu

Current address, R. S. MacKay: DAMTP, Silver Street, University of Cambridge, CB3 9EW, U.K.

E-mail address: R.S.MacKay@damtp.cam.ac.uk 